\title{
Modelling Orthorhombic Anisotropic Effects for Reservoir Fracture Characterization of a Naturally Fractured Tight Carbonate Reservoir, Onshore Texas, USA
}

\author{
Olawale Olakunle Osinowo, ${ }^{1,2,3}$ Mark Chapman, ${ }^{3,4}$ Rebecca Bell, ${ }^{2}$ and Heloise B. Lynn ${ }^{5}$
}

\begin{abstract}
In this study we present a step-by-step theoretical modelling approach, using established seismic wave propagation theories in anisotropic media, to generate unique anisotropic reflection patterns observed from three-dimensional pure-mode pressure (3D-PP), full-azimuth and full-offset seismic reflection data acquired over a naturally fractured tight carbonate field, onshore Texas, USA. Our aim is to gain an insight into the internal structures of the carbonate reservoir responsible for the observed anisotropic reflection patterns. From the generated model we were able to establish that the observed field seismic reflection patterns indicate azimuthal anisotropy in the form of crack induced shearwave splitting and variation in P-wave velocity with offset and azimuth. Amplitude variation with azimuth (AVAZ) analysis also confirmed multi-crack sets induced anisotropy which is characteristic of orthorhombic symmetry, evident as multiple bright and dim-amplitude azimuth directions as well as complete reversal of bright-amplitude to dim-amplitude azimuth direction as the angle of incidence increases from near $\left(\leq 15^{\circ}\right)$ to mid $\left(\geq 30^{\circ}\right)$ offsets. Finally, we fitted the generated P-wave velocity into an ellipse to determine the intensity and orientation (N26E) of the open crack set as well as the direction of the minimum in situ stress axis (N116E) within the reservoir. The derived information served as an aid for the design of horizontal well paths that would intercept open fractures and ensure production optimization of the carbonate reservoir, which was on production decline despite reservoir studies that indicate un-depleted reserves.
\end{abstract}

Key words: Full-offset, crack orientation, azimuthal anisotropy, birefringence, bright and dim azimuth directions.

1 Department of Geology, University of Ibadan, Ibadan, Nigeria. E-mail: wale.osinowo@ui.edu.ng; olawale.osinowo13@alumni.imperial.ac.uk

2 Department of Earth Science and Engineering, Imperial College London, London, UK. E-mail: Rebecca.bell@imperial.ac.uk

3 Edinburgh Anisotropic Project (EAP), Edinburgh, UK.

4 School of Geosciences, The University of Edinburgh, Edinburgh, UK.

5 Lynn Incorporated, La Veta CO 81055, Colorado, USA.

\section{Introduction}

Fractures with horizontal offset or those with small vertical offset $(<1 / 4$ seismic wavelength) are poorly resolved if at all detected on seismic records (Childs et al. 1990). Seismic azimuthal anisotropy analyses offer methods of delineating the orientation and the intensity of these vital structural elements based on P-wave polarization and S-wave splitting in azimuthally anisotropic media, where the shear wave splits into two modes, the fast $\left(\mathrm{S}_{1}\right)$ and slow modes $\left(\mathrm{S}_{2}\right)$ Crampin 1981, 1985; Schoenberg and Sayers 1995; Tsvankin 2005; Grechka et al. 2007). The direction of polarization of the fast mode corresponds to the fracture strike and the delay between $S_{1}$ and $S_{2}$ indicate the degree of anisotropy (Yardley and Crampin 1991). These underlying seismic properties enable indirect mapping of micro-fractures whose detection and characterization offer important information needful for well path design, aiming at intersecting a larger number of permeable fractures, so sweep efficiency and hydrocarbon recovery are improved (Finkbeiner et al. 1997; Bates et al. 1999; Tsvankin and Grechka 2011).

The fundamental theories governing the propagation of seismic waves in anisotropic media with emphasis on velocities and polarization of plane waves have been discussed by Musgrave (1970), Helbig (1994), Schoenberg and Helbig (1997) and others. Crampin (1984) established that most geologic formations possess a certain degree of anisotropy which can be classified into different symmetry classes based on the structure of their elastic stiffness tensors and the number of independent components of the tensors (Thomsen 1986; Tsvankin 2001; Tsvankin and Grechka 2011). Horizontal layering in sedimentary rocks has been 
described to generate layer anisotropy known as vertical transverse isotropy (VTI) (Hornby et al. 1994) while aligned vertical cracks would induce horizontal transverse isotropy (HTI) (Tsvankin and Grechka 2011). The occurrence of set (s) of stressinduced aligned vertical micro cracks within horizontal layered sedimentary formations would combine layer-induced VTI and fracture-imposed HTI to generate orthorhombic anisotropy (Fig. 1). This will presents three mutually orthogonal mirror planes of symmetry (Schoenberg and Helbig 1997), but different principal axes. Orthorhombic symmetry is defined by nine independent elastic tensors, six of which are diagonal and the remaining three off diagonal (Thomsen 2002). The Voigt elastic stiffness matrix for VTI, HTI and Orthorhombic symmetries are presented in Eqs. 1, 2 and 3.

VTI

$C_{m n}=\left[\begin{array}{cccccc}C 11 & C 11-6 C 66 & C 13 & 0 & 0 & 0 \\ C 11-6 C 66 & C 11 & C 13 & 0 & 0 & 0 \\ C 13 & C 13 & C 33 & 0 & 0 & 0 \\ 0 & 0 & 0 & C 55 & 0 & 0 \\ 0 & 0 & 0 & 0 & C 55 & 0 \\ 0 & 0 & 0 & 0 & 0 & C 66\end{array}\right]$

HTI

$C_{m n}=\left[\begin{array}{cccccc}C 11 & C 13 & C 13 & 0 & 0 & 0 \\ C 13 & C 33 & C 33-2 C 44 & 0 & 0 & 0 \\ C 13 & C 33-2 C 44 & C 33 & 0 & 0 & 0 \\ 0 & 0 & 0 & C 44 & 0 & 0 \\ 0 & 0 & 0 & 0 & C 55 & 0 \\ 0 & 0 & 0 & 0 & 0 & C 55\end{array}\right]$

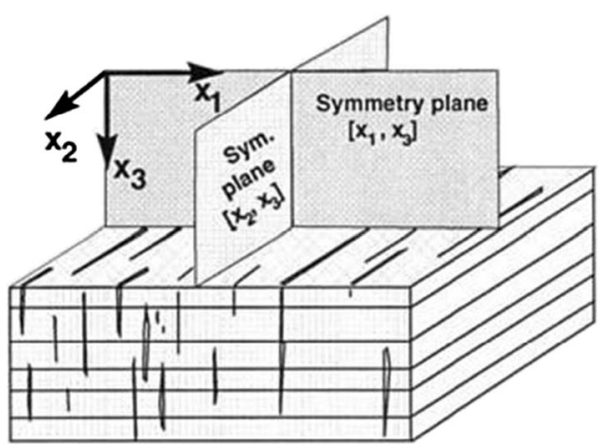

Figure 1

Orthorhombic model due to aligned vertical fractures embedded in horizontally layered medium (Tsvankin 2001)
Orthorhombic

$$
C_{m n}=\left[\begin{array}{cccccc}
C 11 & C 12 & C 13 & 0 & 0 & 0 \\
C 12 & C 22 & C 23 & 0 & 0 & 0 \\
C 13 & C 23 & C 33 & 0 & 0 & 0 \\
0 & 0 & 0 & C 44 & 0 & 0 \\
0 & 0 & 0 & 0 & C 55 & 0 \\
0 & 0 & 0 & 0 & 0 & C 66
\end{array}\right]
$$

An oil-producing field located onshore western Texas, in the United States of America which has been producing from a naturally fractured, less porous and permeable tight carbonate reservoir has recently been experiencing production decline despite reservoir analyses that suggested that the reserve is un-depleted. The field production history also indicates economically sustainable yield from some wells which were thought to have intersected networks of high-density macro-cracks. This background information led to an initial study aimed at delineating the high-density fracture network as well as determining the orientation of the aligned macro-cracks for the purpose of designing horizontal wells that will intersect most of them.

This study presents the step-by-step modelling approach adopted to establish the occurrence, orientation and density of the stress-induced cracks in the carbonate reservoir using the various established rock physics models which indicate seismic wave propagation patterns in anisotropic media. First, the procedure for generating background isotropic models for the shale cap rock and the underlying carbonate reservoir using P- \& S-wave velocities and densities derived from the specially processed azimuth sensitive data is presented. Thereafter, the processes of building in layer-induced VTI into the background isotropic shale and carbonate models using the Thomsen anisotropic parameters obtained by inverting the field data is also highlighted. The gradual injection of two sets of stress-induced aligned vertical penny-shaped cracks of different orientations, densities and aspect ratios into the already established VTI carbonate model using Hudson classic equivalent medium theory was conducted and the resulting azimuth sensitive seismic reflection patterns after fluid injection using Brown and Korringa model are presented). 


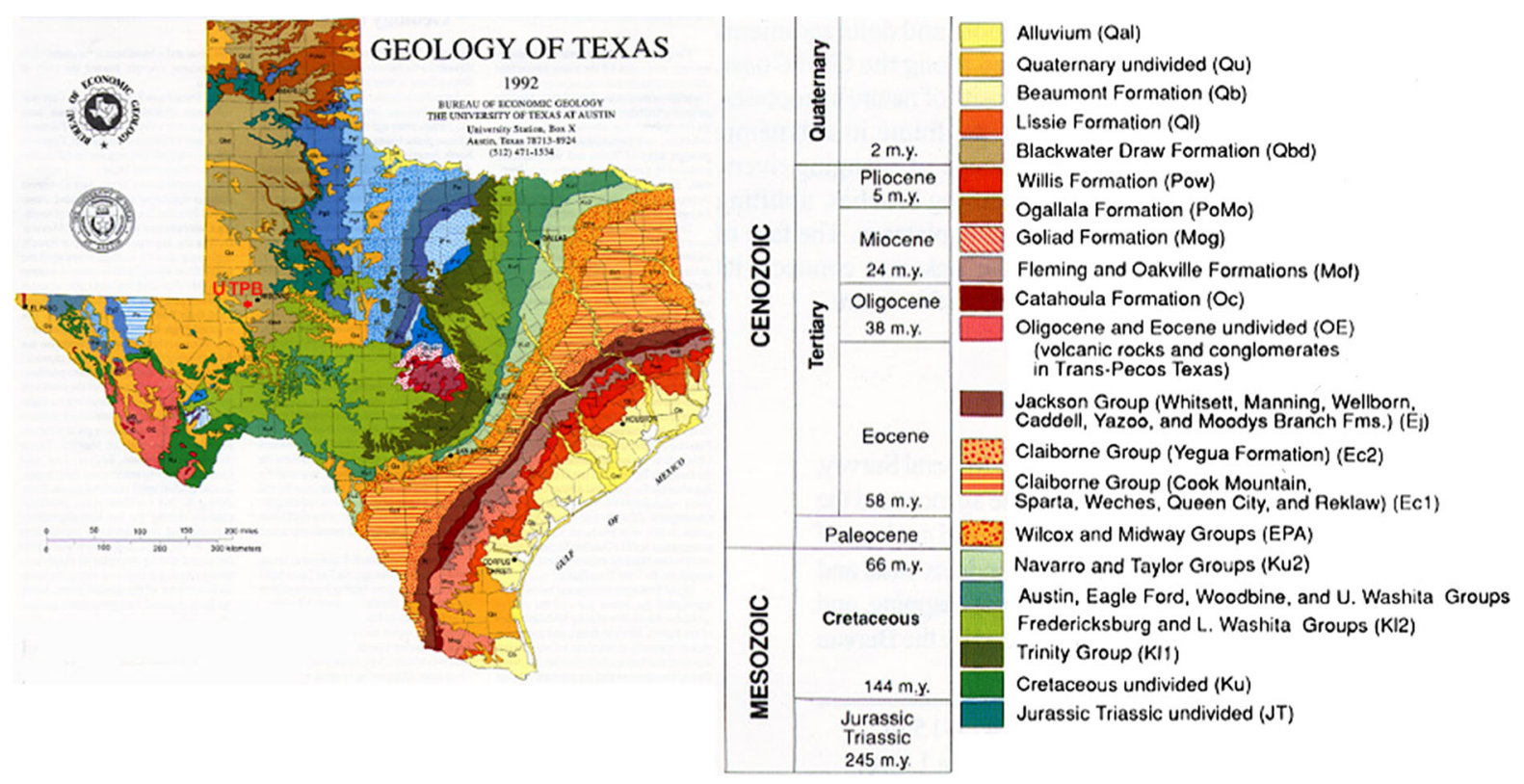

Figure 2

Geological Map of Texas State (Source: Bureau of Economic Geology, The University of Texas at Austin; www.beg.utexas.edu 2016)

\section{Geology of the Study Area}

The study area is located in the Texas arm of the Permian Basin which consists of Delaware, Central Basin Platform and Midland sub-basins. It is an onshore field situated in the western part of Texas in the United States of America (Fig. 2). The geological history of Texas is recorded in different rock exposures scattered throughout the state; these records indicate that Texas has undergone a long and dynamic geological evolution, which includes magmatic episodes, structural deformation, and sedimentary processes (Ross 1970). Different orogenic events, including those associated with the collision and separation of the North American tectonic plate and the African-South American plates as well as some mountain building episodes (Grenville orogeny of eastern North America) helped to shape and generate many structural features (Dawin 1991). These features include buried basins, structural highs and sedimentary accumulations that host Texas' largest hydrocarbon reserves (Darwin 1991). The different tectonic events also resulted in a series of structural deformation of the North American continental plate around the state of Texas and environs.
The structural deformation generated basin subsidence, rifting, crustal uplift, thrusting, fold belts, mountain ranges and other structural signatures seen throughout the state (Adams 2003; Montgomery et al. 2005). Figure 2 is a geological map of Texas showing different geological events within the state.

Different potential sources of hydrocarbon generation exist within the thick sedimentary units deposited during the Paleozoic to recent; the prominent source is the organic rich calcareous and shaly limestone. The shale known as the Eagle Ford Shale in some of the basins is the main source of hydrocarbon generation in most part of Texas. The shale is

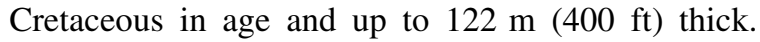
Some parts of the shale units contain up to $70 \%$ carbonate content (Ball 1995). Oil and natural gas have been found to be hosted in a variety of rocks within the state. Ball (1995) indicated that the reservoir rocks within the basin are usually porous limestone, dolomite, dolomitized mudstone and wackestone, and smaller amounts of fine-grained clastics which are frequently associated with evaporates. The carbonate rocks were deposited on platforms and at platform margins, and are naturally of low porosity and permeability. However, reservoir 


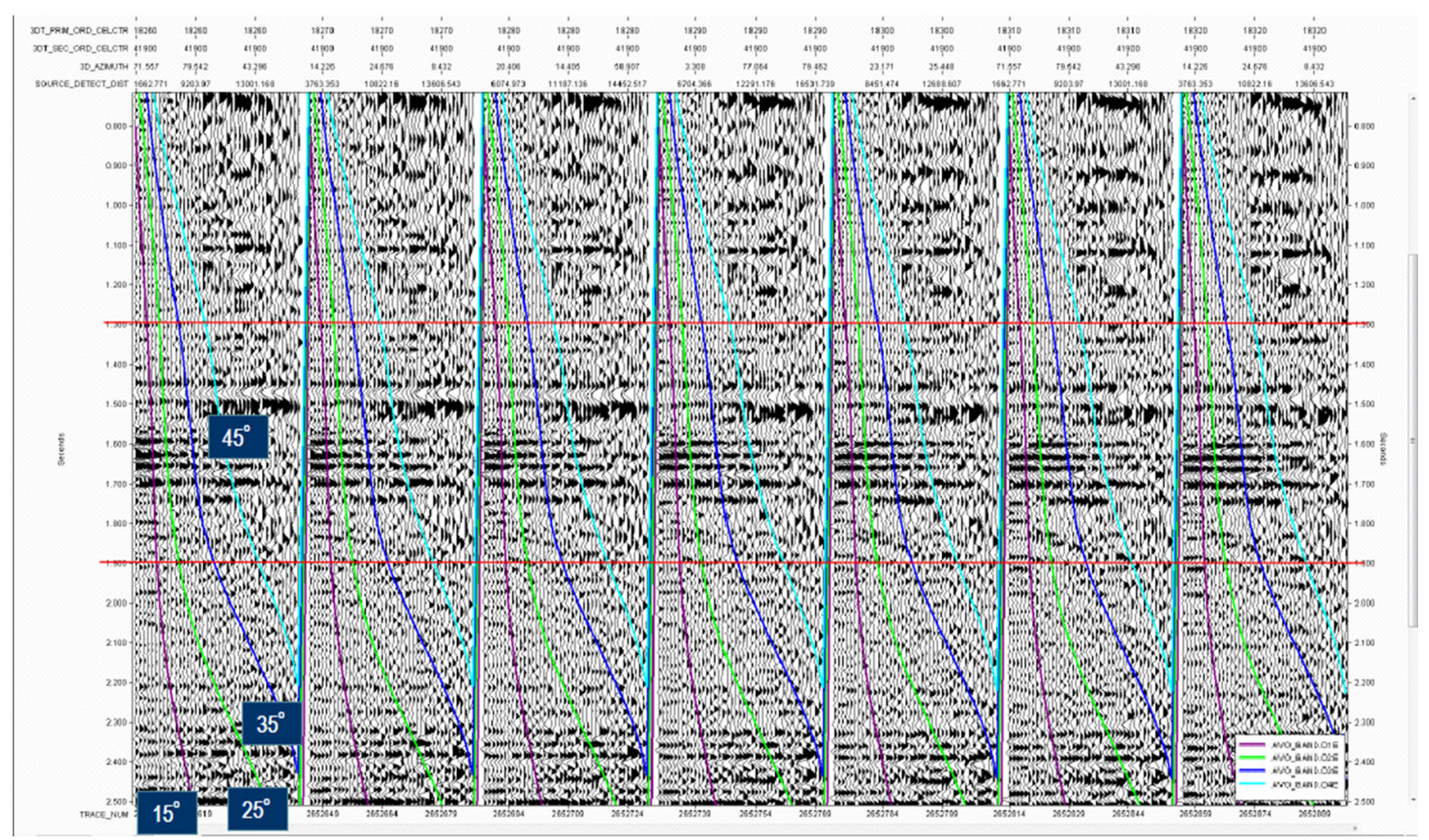

Figure 3

Offset vector tile (OVT) Prestack depth migrated PP- gathers

quality is enhanced by fracturing, dissolution and selective dolomitization which increased porosity and permeability to $12 \%$ and 18 millidarcies, respectively (Ball 1995).

\section{Data Acquisition and Processing Techniques for Anisotropy Preservation}

The initial investigations led to the acquisition of new sets of full-azimuth full-offset 3D PP seismic data by WesternGeco. The data were acquired using a vibroseis source and WesternGeco UniQ integrated point-receiver Geophone Accelerometers (GAC). The receivers consisted of 1-C accelerometers which were laid in parallel lines at a line spacing of $300 \mathrm{~m}$ and receiver points spacing of $6 \mathrm{~m}$. The source lines were $370 \mathrm{~m}$ apart with $60 \mathrm{~m}$ spacing separation. The source-receiver pattern enabled the acquisition of symmetric, full fold, long-offset azimuthal data capable of preserving both transverse and azimuthal isotropy necessary for anisotropic analysis in this study.
The acquired data were specially processed using offset-vector-tile azimuthal Prestack depth migration (OVT-PSMD) to preserve velocity variation with azimuth as well as other azimuthal anisotropic information (Calvert et al. 2008). This is essential because the convectional time migration method assumes an isotropic symmetric media where velocity is invariant with orientation. Thus the Taylor series expansion of the ray-traced two-way travel time is truncated after the second-order term to estimate the hyperbolic P-wave normal-moveout (NMO) (Taner and Koehler 1969). However, while this approximation is sufficient to handle the hyperbolic moveout equation for short offsets of a homogeneous isotropic plane layer with zero dip and offset-depth ratio $<1$, it is insufficient for anisotropic media and long offset data with offset-depth ratio $>1$, such as required for this study (Alkhalifah and Tsvankin 1995; Tsvankin and Thomsen 1994).

Finally, the processed data (Fig. 3) were inverted to extract parameters such as interval velocities ( $V_{\text {INT-FAST }}$ and $V_{\text {INT-SLOW }}$ ) in the fast and slow 
directions, azimuth of the fast and slow directions, interval anisotropy, percentage anisotropy, Thomsen anisotropic parameters and other information necessary to achieve the study's objectives.

\section{Methods}

The direct interpretation of anisotropy from seismic data is difficult because defining the nine independent orthorhombic elastic constants is challenging. An indirect method of defining the Earth model which generated the observed seismic responses offers a relatively simpler method of obtaining credible information about the crack network, orientation and density of a naturally fractured reservoir. This approach involves establishing the background transverse isotropic model and thereafter iteratively updating the model until the azimuthal anisotropy observed in the field data is obtained. The modelling exercise was performed in three stages. First, background isotropic Earth models for the shale cap rock and the underlying carbonate reservoirs were constructed. Then layer-induced VTI was built into the background models using the Thomsen (1995) anisotropic parameters. Dry aligned vertical penny-shaped ellipsoid cracks sets were injected into the carbonate reservoir model to generate orthorhombic symmetry. Finally, the dry crack sets were saturated with formation fluid in order to model the field scenario. Several existing rock physics models capable of generating elastic stiffness tensors for both transverse and azimuthal anisotropy were employed using rock unix (RU) codes developed by the Edinburgh Anisotropy Project (EAP) unit of the British Geological Survey (BGS) in Edinburgh. The RU code was developed from existing basic rock physics theories and it runs on Seismic Unix (SU) in a Linux environment (Stockwell and Cohen 2008).

\subsection{VTI Models for Shale and Carbonate Reservoirs}

Generating VTI models involves the computation of the elastic tensors of the layered media, which reflects VTI anisotropy. The initial isotropic model for the shale and carbonate layers were generated from the vertical P-wave velocity $\left(V_{\mathrm{p}^{-}}\right.$ shale $=4.176 \mathrm{~km} / \mathrm{s}, \quad V_{\mathrm{p}}$-carbonate $\left.=5.852 \mathrm{~km} / \mathrm{s}\right)$, the vertical S-wave velocity $\left(V_{\mathrm{s}}\right.$-shale $=2.53 \mathrm{~km} / \mathrm{s}$, $V_{\mathrm{s}}$-carbonate $\left.=3.292 \mathrm{~km} / \mathrm{s}\right)$ and density $\left(\rho_{\text {sha- }}\right.$ le $\left.=2500 \mathrm{~kg} / \mathrm{m}^{3}, \quad \rho_{\text {carbonate }}=2610 \mathrm{~kg} / \mathrm{m}^{3}\right) . \quad$ The parameters were determined from azimuthally isotropic prestack elastic inversion of the field seismic record. For the isotropic starting models, the Thomsen parameters epsilon $(\varepsilon)$, delta $(\delta)$ and gamma $(\gamma)$ were set to zero $(\varepsilon, \gamma, \delta=0)$. The obtained elastic stiffness tensors have the form $C_{11}=C_{22}=C_{33}$ and $C_{44}={ }_{55}=C_{66}$.

Subsequently, vertical transverse anisotropy was introduced into the isotropic models by incorporating the field measured Thomsen VTI parameters for both the shale and carbonate layers. The VTI Thomsen parameters $0.1,0.075,0.12$ and $0.013,0.012$ and 0.05 representing the epsilon $(\varepsilon)$, delta $(\delta)$ and gamma $(\gamma)$ values for the shale unit and the carbonate rock modified the isotropic elastic symmetry to a form where $C_{11}=C_{22}>C_{33}$ and $C_{44}={ }_{55}>C_{66}$.

\subsection{Orthorhombic Model for Carbonate Reservoirs}

The VTI models were used as background models upon which sets of aligned vertical cracks were injected to create the orthorhombic anisotropy, suspected to be responsible for the observed seismic attributes extracted from the field data acquired over the carbonate field. The a priori geological information for the basin as well as other analytical studies suggests two sets of aligned vertical cracks (aligned along NE direction) in the carbonate reservoir (Friedman and Wiltschko 1992). The cracks are believed to be induced along the principal axes of the regional paleo tectonic stress fields that acted on the sedimentary basin. The Schoenberg (1980) linear-slip theory was employed to generate the effective azimuthal isotropic medium for the carbonate reservoir, while the Hudson (1981) penny-shaped crack model was used to inject vertical ellipsoidal shaped cracks of crack density $\left(C_{\mathrm{d}}\right)$ into the background model. The crack density is an expression of the number of cracks per unit volume, multiplied by the cube of the crack's radius:

$$
C_{\mathrm{d}}=\frac{N}{V} r^{3}=\frac{3 \emptyset_{\mathrm{C}}}{4 \pi \alpha},
$$


where $\frac{N}{V}$ is the number of cracks per unit volume, $r$ is the crack radius, $\alpha$ is the crack aspect ratio and $\emptyset_{C}$ is the crack porosity. The aspect ratio, a measure of the degree of crack thinness/fatness, is the ratio of the major and minor radii of the penny-shaped crack.

Two sets of aligned vertical penny-shaped cracks (one micro-crack aligned along N95E direction and the second macro-crack in N20E direction), having different crack densities and aspect ratios $\left(C_{\mathrm{d}}=0.003 \& 0.1, \alpha=0.001 \& 0.01\right.$, respectively), were injected into the VTI background model. The crack orientations, crack densities and aspect ratios of the two crack sets were derived through painstaking and careful trial of different parameters, until the theoretical modelled anisotropy matches that observed in the field. The modelling operation was constrained by the degree of measured anisotropy of $0.037(3.7 \%)$ at $45^{\circ}$ angle of incidence from the field record. Thomsen (1995) model was used to compute the elastic stiffness tensors for the generated medium where the initial tensor distribution in the VTI medium changes the form where $C_{11}=C_{22}>C_{33}$ and $C_{44}={ }_{55}>C_{66}$, into an orthorhombic case where $C_{11} \neq C_{22} \neq C_{33}$ and $C_{44} \neq C_{55} \neq C_{66}$.

\subsection{Fluid Injection}

The defined orthorhombic model up until now was a rock matrix with dry crack sets; however, in order to simulate the field scenario as closly as possible, large, high crack density fractures (N20E) were injected with brine, oil and gas using the Brown and Korringa (1975) fluid substitution theory expressed in Eq. 5:

$$
\left(C_{\mathrm{sat}}-C_{\mathrm{s}}\right)^{-1}=\left(C_{\mathrm{dry}}-C_{\mathrm{s}}\right)^{-1}+\left[\emptyset\left(\mathrm{C}_{\mathrm{fl}}-\mathrm{C}_{\emptyset \mathrm{s}}\right)\right]^{-1},
$$

where compressibilities $\quad C_{\mathrm{sat}}=\frac{1}{K_{\mathrm{sat}}}, \quad C_{\mathrm{dry}}=\frac{1}{K_{\mathrm{dry}}}$, $C_{\mathrm{s}}=\frac{1}{K_{\mathrm{s}}}$ and $C_{\emptyset \mathrm{s}}=\frac{1}{K_{\emptyset \mathrm{s}}}$. For a homogeneous mineral phase approximation, $K_{\mathrm{s}}=K_{\emptyset \mathrm{s}}=K_{\text {mineral }}$ and $C_{\mathrm{s}}=C_{\emptyset \mathrm{s}}=C_{\text {mineral }}=\frac{1}{K_{\text {mineral }}} . K_{\text {mineral }}$ is the bulk modulus of the mineral and $K_{\mathrm{fl}}$ is bulk modulus of the fluid.

Figure 4 presents diagrams illustrating the different modelling steps taken to build the seismic reflection attributes defining the orthorhombic anisotropy in the field data.

\section{Interpretation and Discussion of Results}

A geological model consisting of a fine-layered shale cap rock and an underlying naturally fractured carbonate reservoirs was generated. Some angle-dependent and azimuth-varying seismic attributes such as $\mathrm{P}$ - and $\mathrm{S}$-wave velocities (in the individual modelled geological units) and the seismic reflection amplitude at the shale-carbonate interface were computed and presented below.

Upon the introduction of the Thomsen anisotropic parameters for the purpose of creating VTI media, two types of velocity anisotropy became evident. The first is that both the $\mathrm{P}$ - and $\mathrm{S}$-waves became angle dependent as the velocities now vary with angle of incidence (Figs. 5, 6). The second noticed anisotropic effect is that the $\mathrm{S}$-wave splits into two $\left(V_{\mathrm{s} 1} \& V_{\mathrm{s} 2}\right)$. The plot of $V_{\mathrm{p}}, V_{\mathrm{s}}$ versus incident angle for shale (Fig. 6) indicates an increase in P-wave velocity $(4.17-4.58 \mathrm{~km} / \mathrm{s})$.

The splitting of S-wave into two orthogonal polarizations in a transversely anisotropic medium is a typical characteristic splitting described by Crampin and Peacock (2008) and it is caused by finelayering in stratified formations, where polarization is controlled by the faster direction of wave propagation. S-wave splits into $S_{\mathrm{V}}$ and $S_{\mathrm{H}}$ with vertically polarized part of the wave $\left(S_{\mathrm{V}}\right)$ travelling faster within the layers, while $S_{\mathrm{H}}$ travels slower across the fine thin sedimentary layers (Crampin and Peacock 2008). However, the observed fine-layering-induced S-wave polarization does not reflect azimuthal anisotropy as both the $V_{\mathrm{p}}$ and $V_{\mathrm{s}}\left(V_{\mathrm{s} 1} \& V_{\mathrm{s} 2}\right)$ are azimuthally invariant.

The introduction of aligned vertical cracks into the carbonate reservoir generated azimuth-dependent anisotropy, which exhibits two kinds of seismic Pand S-waves' anisotropy. The first anisotropic effect is that $\mathrm{P}$ - and $\mathrm{S}$-waves became azimuth dependent, here P- and S-waves' velocities vary at different azimuth directions unlike in layer-induced transverse anisotropy (Figs. 7, 8 and 9), where both velocities (P $\&$ S) remain constant at all azimuthal directions. The 


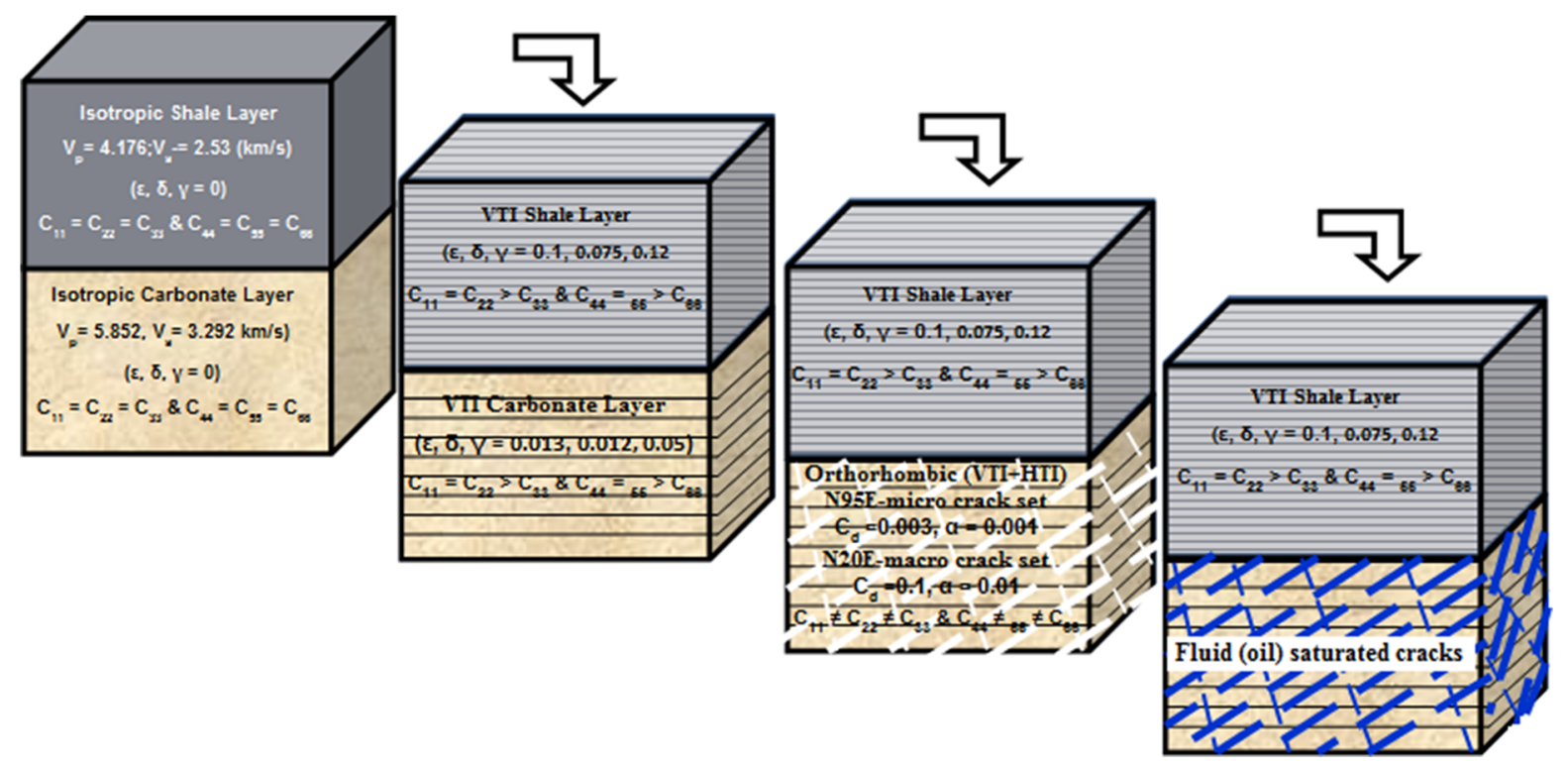

Figure 4

Diagrams illustrating stepwise modelling procedure to generate orthorhombic anisotropy in the carbonate reservoir

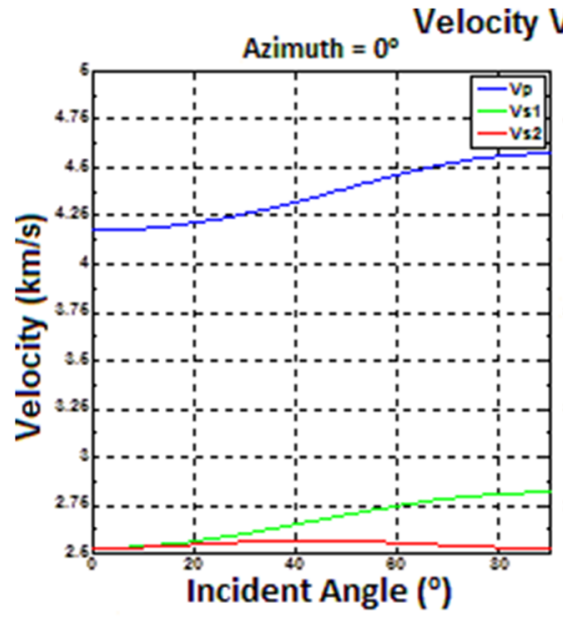

Incident Angle $\left({ }^{\circ}\right)$

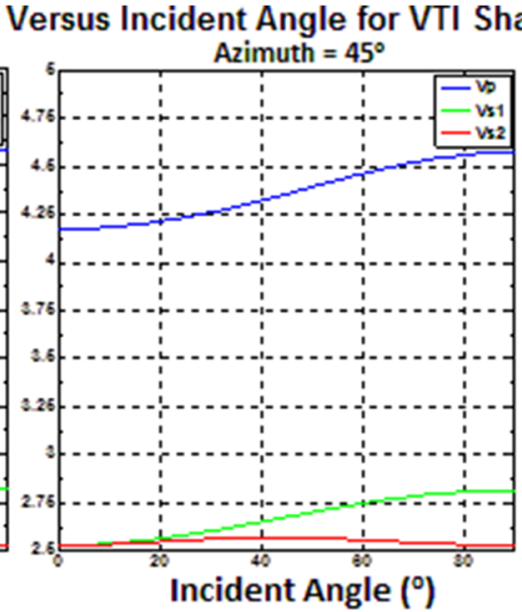

Figure 5

Velocity variation with Angle of Incidence for VTI medium (shale) at $0^{\circ}, 45^{\circ}$ and $180^{\circ}$ azimuth directions

observed anisotropic effect due to aligned vertical crack is azimuthally aligned shear wave splitting which agrees with Crampin (1989) suggestion that the degree of P-wave anisotropy depends on the orientation of parallel vertical micro-cracks, crack density and to a small extent, changes in crack aspect-ratio.

The polar plots of azimuthal variation with velocities $\left(V_{\mathrm{p}}, V_{\mathrm{s} 1}\right.$ and $\left.V_{\mathrm{s} 2}\right)$ are presented in Figs. 8a- $\mathrm{d}$ and $9 \mathrm{a}-\mathrm{d}$, respectively. Azimuthal velocity variations are caused by vertical fracture sets oriented N95E and N20E. The plots indicate no significant azimuthal anisotropy effect at zero offset distance, as all the velocity components remain constant with azimuth. Small degree of azimuthal variation is observed at the near offsets and this gradually increases as the offset angle increases. The azimuthal anisotropic effect is more significant in the 


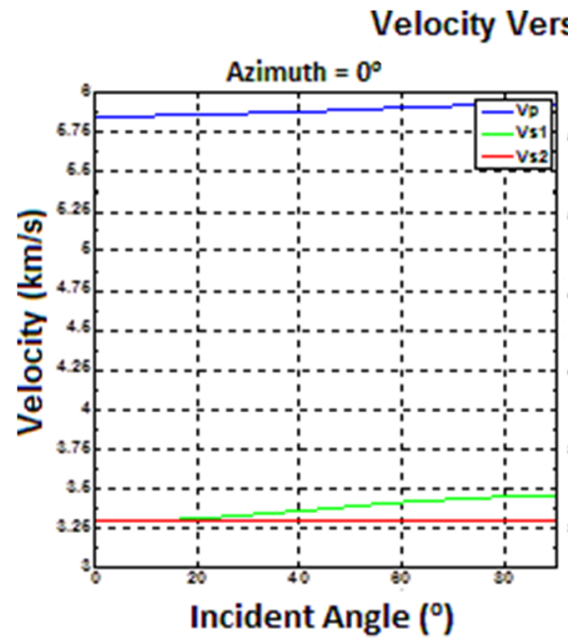

Incident Angle $\left({ }^{\circ}\right)$

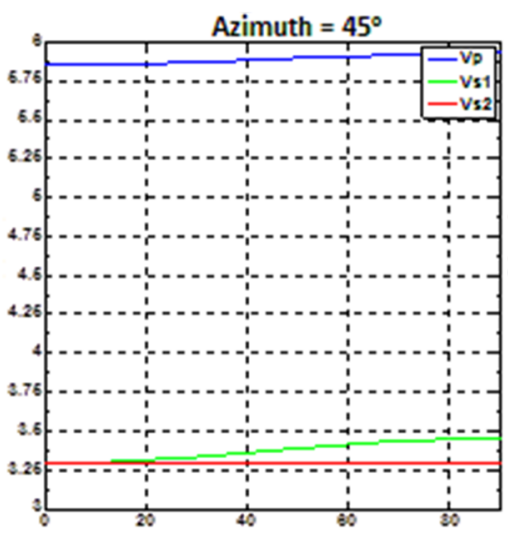

Incident Angle $\left({ }^{\circ}\right)$
Azimuth $=180^{\circ}$

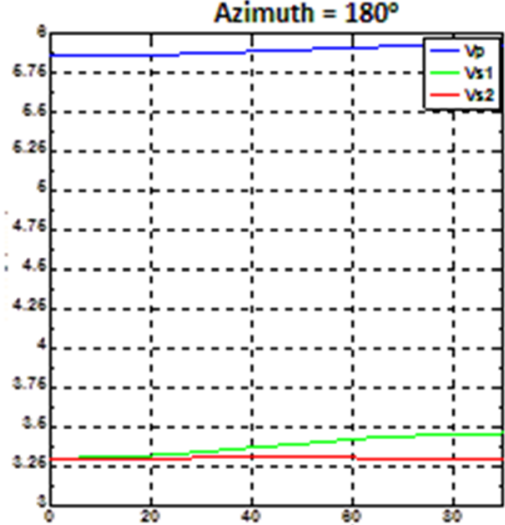

Incident Angle ( ${ }^{\circ}$ )

Figure 6

Velocity versus angle of incidence for VTI medium (carbonate reservoir) at $0^{\circ}, 45^{\circ}$ and $180^{\circ}$ azimuth directions

Polar Plot of Velocity Variation with Azimuth due Azimuthal Anisotropic (VTI+HTI) in Carbonate Layer (Crack $=$ N95E)

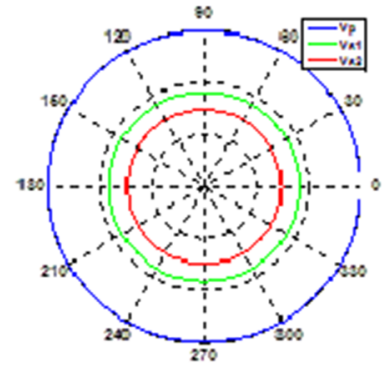

Inc. Angle $=0^{\circ}$

(a)

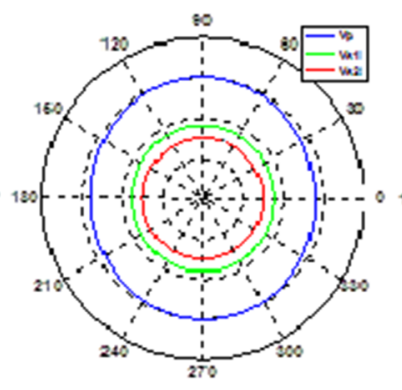

Inc. Angle $=30^{\circ}$

(b)

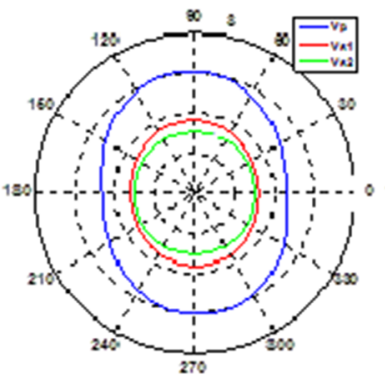

Inc. Angle $=60^{\circ}$

(c)

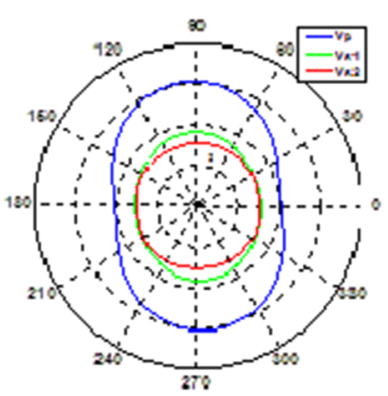

Inc. Angle $=90^{\circ}$

(d)

Figure 7

Polar plots of azimuthal variation in velocities $\left(V_{\mathrm{p}}, V_{\mathrm{s} 1}\right.$ and $\left.V_{\mathrm{s} 2}\right)$ due to vertical aligned crack set N95E

compressional wave than for the shear wave. Another very visible effect in the polar plots is that the velocity ellipse is aligned along the crack orientation where the maximum velocity is observed parallel to the crack axis. This agrees with the observation recorded by Hsu and Schoenberg (1993) and Hudson (1981) that during polarization in an anisotropic medium, the fastest P-wave velocity travels in a direction parallel to the orientation of the aligned fractures.

Figure 9 expresses the effect of fracture-induced anisotropy (azimuthal anisotropy) in the form of variation in P- and S-waves' velocity with angle and $\mathrm{S}$ waves birefringence.

The crack-induced azimuthal S-wave splitting is different from $\mathrm{S}$-wave splitting induced by fine layering. While $V_{\mathrm{s} 1}$ and $V_{\mathrm{s} 2} \mathrm{~S}$-wave splitting caused by the layering remains invariant with azimuth, the velocities of slow and fast S-waves due to azimuthally aligned shear wave splitting vary for measurements taken at different azimuths. In addition, there is also splitting of S-wave at zero and very low angle of incidence $\left(<5^{\circ}\right)$, which did not characterise layer-induced S-wave splitting. Therefore, this 
Polar Plot of Velocity Variation with Azimuth due to Azimuthal Anisotropy (VTI+HTI) For Carbonate Layer $($ Crack $=$ N20E)

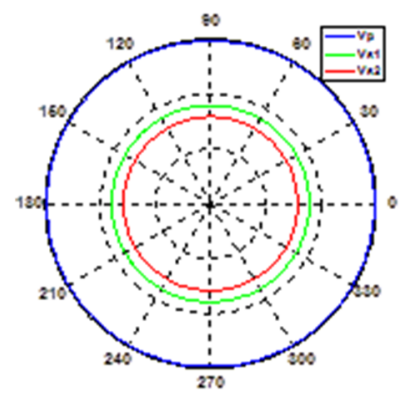

Inc. Angle $=0^{\circ}$

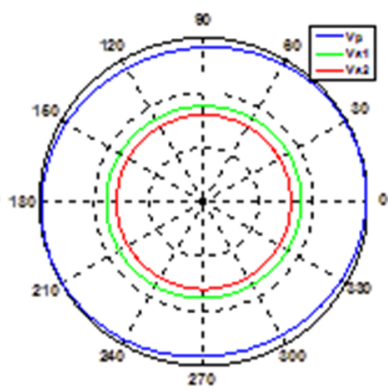

Inc. Angle $=30^{\circ}$

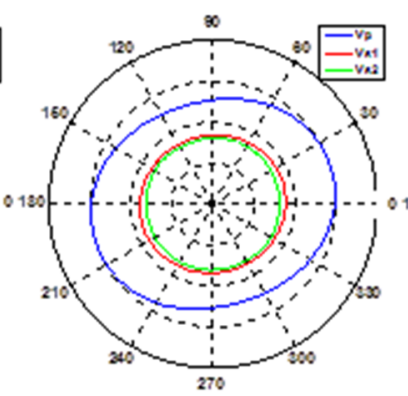

Inc. Angle $=60^{\circ}$

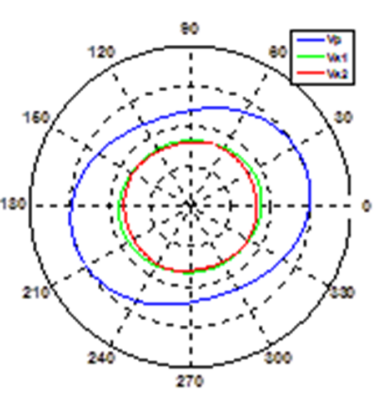

Inc. Angle $=90^{\circ}$

Figure 8

Polar plots of azimuthal variation in velocities $\left(V_{\mathrm{p}}, V_{\mathrm{s} 1}\right.$ and $\left.V_{\mathrm{s} 2}\right)$ due vertical aligned crack $\mathrm{N} 20 \mathrm{E}$

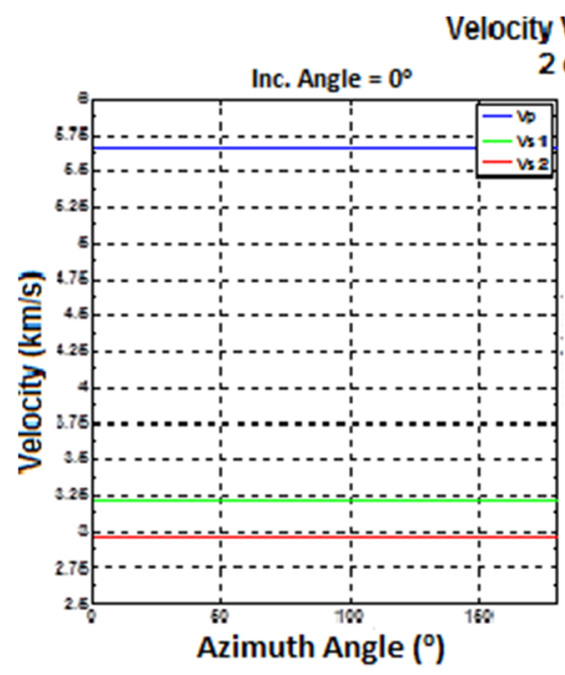

(a)

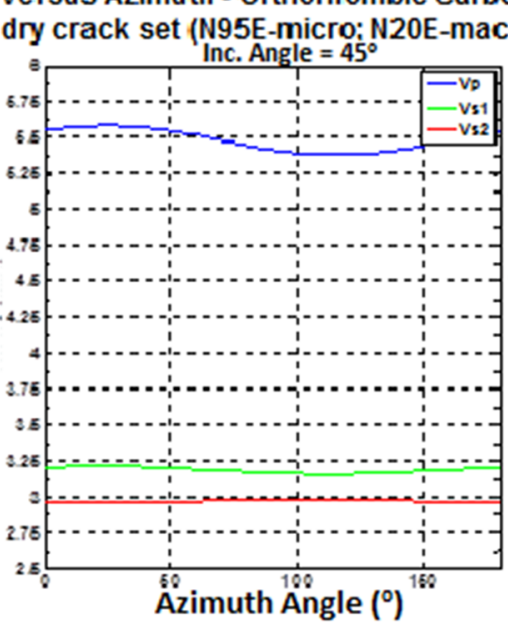

(b)
Inc. Angle $=180^{\circ}$

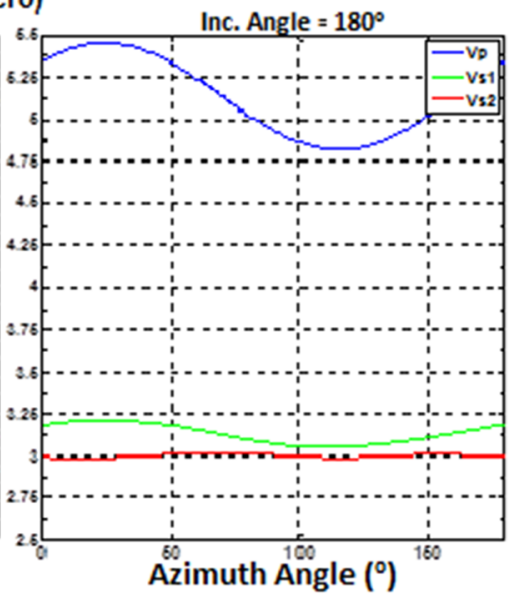

(c)

Figure 9

Velocity versus azimuth for orthorhombic carbonate medium (two dry cracks sets) at $0^{\circ}, 45^{\circ}$ and $180^{\circ}$ incident angles

suggests that the splitting is not layer-induced but crack-related. The azimuthally aligned shear wave splitting occurs when shear wave propagates through a medium that includes stress-aligned parallel vertical micro-cracks, which are highly compliant to small changes in stress (Crampin and Peacock 2008). The two split shear waves $\left(S_{1}\right.$ and $\left.S_{2}\right)$ are both vertically propagating (Lynn and Thomsen 1990). $S_{1}$ is polarized parallel to the fracture and travels at a velocity similar to what would be observed in an isotropic medium (Lynn and Thomsen 1990) and thus faster than $S_{2}$ which is polarized perpendicular to the fracture set orientation and delayed by fractures. Wild and Crampin (1991) defined crack-induced anisotropy as extensive dilatancy anisotropy (EDA), when combined with the layer-induced or lithologic anisotropy that yields an orthorhombic anisotropic symmetry with three mutually perpendicular planes of symmetry (Bush and Crampin 1991).

The degree of $\mathrm{P}$-wave anisotropy due to different crack densities $\left(C_{\mathrm{d}}=0.001,0.01,0.1\right.$ and 0.2$)$ in the modelled carbonate reservoir as observed in Fig. 10a, $\mathrm{b}$ is significant, recorded as azimuthal variation in P-wave velocity and has an increasing relationship 
with the crack density. The degree of observed P-wave anisotropy is highest for the model with highest crack density. This is related to the fact that the presence of aligned fractures/cracks naturally makes a medium more compliant (Schoenberg and Helbig 1997); hence, cracks with very small crack density will introduce small compliance. However, high crack density will introduce large amount of preferentially oriented compliance, which will result in azimuthal velocity anisotropy.

Aspect ratio, which measures the degree of fatness/thinness of the crack, shows little or no significant effect at low crack densities and very small anisotropic effect in both the P- and S-waves, even at high crack density (Fig. 11). The polar plots of azimuthal variation of $\mathrm{P}$ - and $\mathrm{S}$-wave's velocities with aspect ratio indicate no significant variation in velocity with azimuth at low crack densities; no significant azimuthal variation effect was noticed with increasing aspect ratio from 0.001 to 0.2 at low crack density (crack density $\leq 0.01$ ). However, at higher crack densities, $(\geq 0.1)$ the effect of increasing aspect ratios became slightly noticeable (Fig. 11b). It is worth to note here that the azimuthal contribution of crack's aspect ratio to azimuthal anisotropy is small even at high crack density, recorded as very small variation in P-wave anisotropy at aspect ratio of 0.001 compared to that at 0.2 .

The insignificant effect of varying aspect ratio on azimuthal anisotropy as explained by Thomsen (1995) is due to the insensitivity of crack compliance to aspect ratio at low frequency. This modelling experiment is a low frequency experiment and assumed crack scale length that is less than the seismic wavelength. At low frequency, there is enough time for fluid to equilibrate locally between adjacent cracks and pores (Chapman 2003; Mavko and Jizba 1991); this implies that seismic waves will cause flow of fluid across interfaces (squirt flow) from compliant into less compliant/stiffer adjacent cracks and pores, especially in non-isolated cracks (Muller et al. 2010). This will make the effect of different aspect ratios insignificant. At high frequency, however, there is no time for squirt flow and fluid equilibration, which implies that the effect of fat or thin cracks will be more visible (Mavko and Jizba 1991; Dvorkin et al. 1994, 1995; Chapman 2003).

Saturating the cracks with fluid (brine, oil and gas) contributes significantly to the azimuthal anisotropic effect of P-wave velocity (Fig. 12); however, the highest azimuthal effect was recorded with gas. This is because a compliant empty isolated crack will be

\section{Velocity Variation with Azimuth at Different Crack Densities}

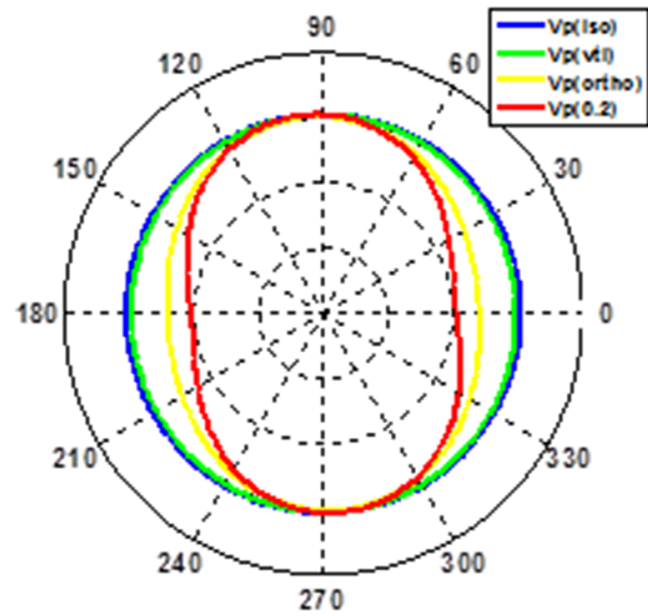

(Crack $=$ N95E)

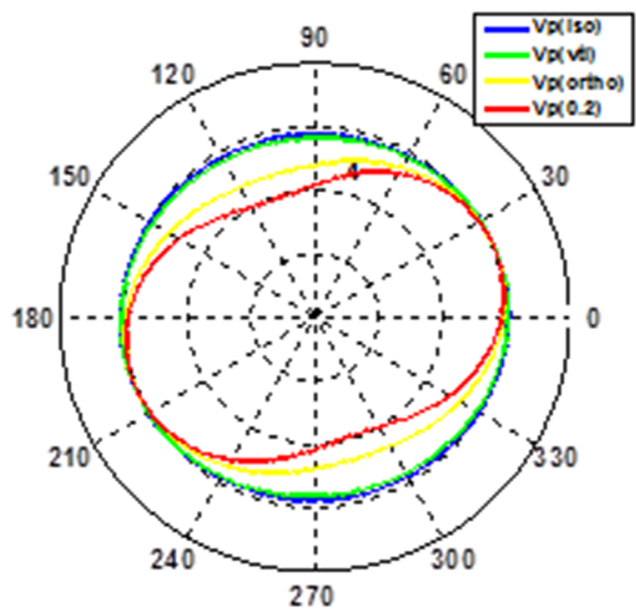

(Crack $=$ N20E)

Figure 10

a, b Polar plots of azimuthal variation in velocity $\left(V_{\mathrm{p}}\right)$ with crack density of aligned vertical cracks N95E and N20E, respectively 


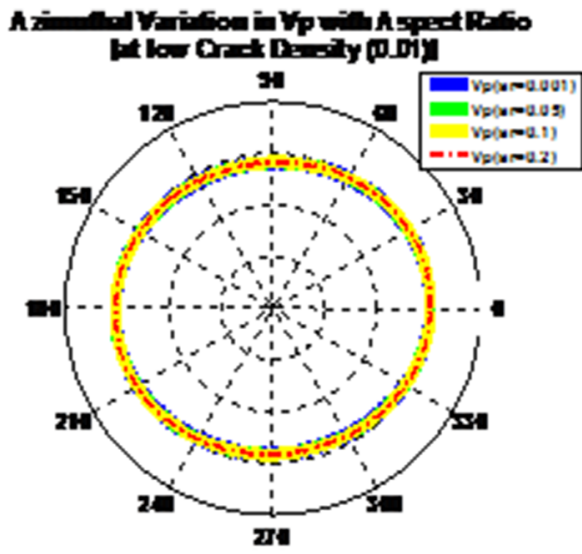

(a)

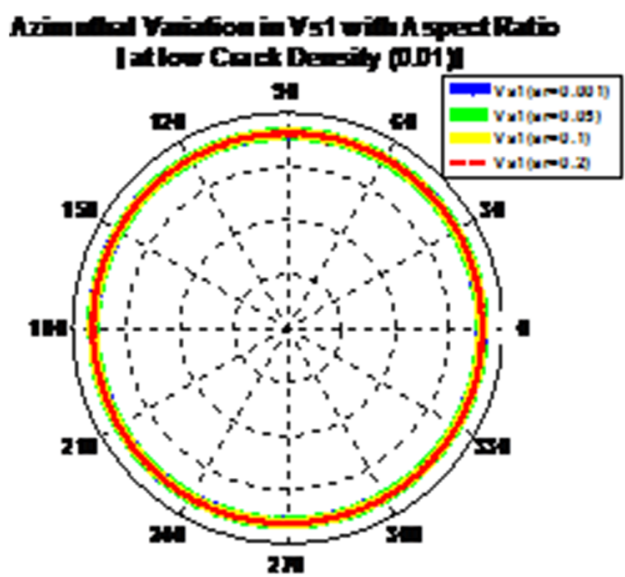

(c)

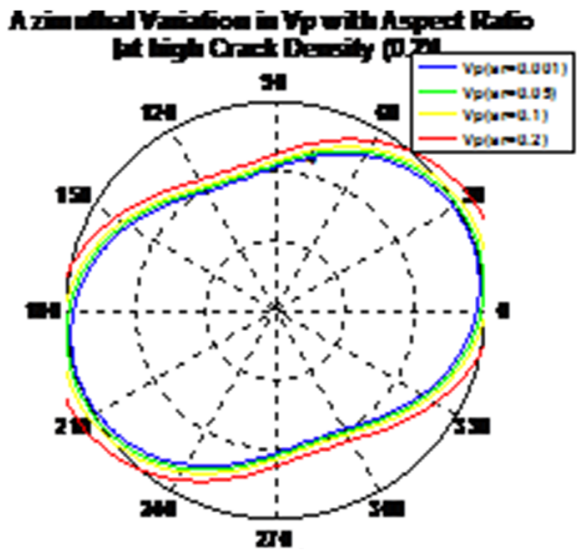

(b)

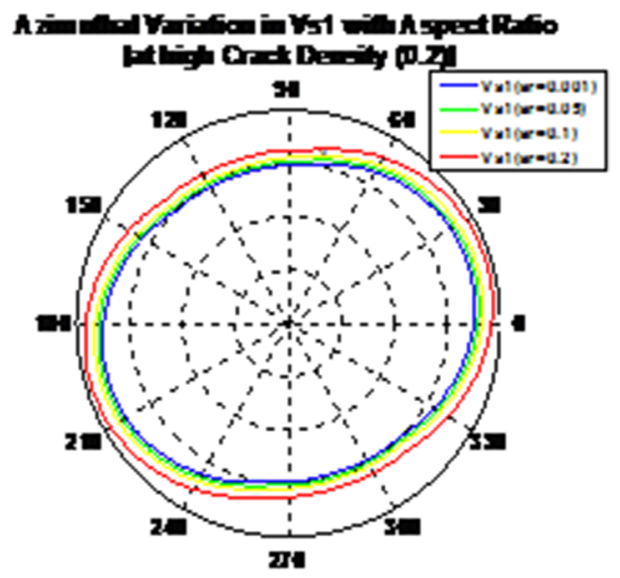

(d)

Figure 11

a-d Polar plots of azimuthal variation in velocities $\left(V_{\mathrm{p}} \& V_{\mathrm{s} 1}\right)$ with aspect ratio at low and high crack densities $(\mathrm{N} 20 \mathrm{E})$

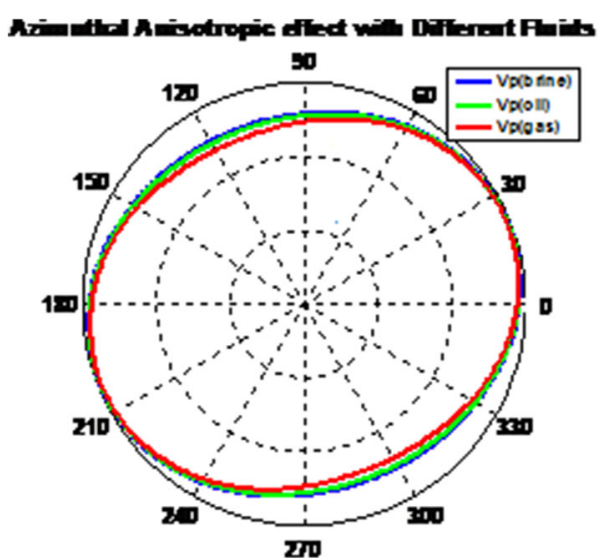

Figure 12

Polar plot of azimuthal variation with different fluid types stiffened by incompressible fluid and this will make the medium stiffer (Thomsen 1995). For connected cracks, however, especially if connected to less compliant equant pore spaces, P-wave propagation will cause the fluid to squirt into adjacent pore spaces and thus make the cracks less compliant. This is responsible for P-wave anisotropy due to fluid saturation. Since gas is the most compressible fluid of the three, the effect is significant for both isolated and connected cracks.

A very subtle but visible difference in the degree of anisotropy as a result of injecting the cracks with oil (Fig. 13) is visible, especially when compared with the dry crack anisotropy effect. Also the degree of anisotropy observed in the resultant oil-saturated 
model is approximately equal to that recorded by the field data presented in Fig. 3.

Amplitude Variation with Angle analysis results obtained using the Aki and Richards' modified/simplified form of Zeoppritz equation (Aki and Richards 1980) is presented in Fig. 14a-c indicating a subtle variation in P-wave reflection coefficient (Rpp) with angle of incidence. This variation in the form of a slight reduction in Rpp from zero offset to near, mid and far mid offsets (around $40^{\circ}$ angle of incidence) causes a dimming of amplitude with offset, which is a case of class I AVO/AVA defined by Rutherford and Williams (1989).

\subsection{Amplitude Variation with Azimuth (AVAZ)}

Azimuthal variation in seismic reflection amplitude is apt at establishing the presence of aligned vertical cracks (Crampin 1993), thus very useful in identifying HTI, orthorhombic and other higher symmetric media. The AVAZ results indicate significant variation in Rpp with azimuth and incident angle. Figure $15 \mathrm{a}-\mathrm{f}$ presents the plots of seismic reflection amplitude at $7^{\circ}, 15^{\circ}, 30^{\circ}, 45^{\circ}, 75^{\circ}$ and $90^{\circ}$ against azimuth. The recorded variation in amplitude with azimuth is a reflection of azimuthal anisotropy due to the presence of stress-induced aligned vertical cracks (Crampin 1993). However, for HTI with a single set of aligned vertical cracks, the variation is expected to be steady in the form of a stable increase or reduction (depending on crack orientation) in amplitude with azimuth, which predicts largest azimuthal variation at far offset angles and expected to decrease with azimuth (Lynn 2014; Sayers 2002). The variation in amplitude as shown in Fig. 16a-f is not steady; the amplitude value rather increases or decreases depending on azimuthal direction.

This pattern of seismic reflection amplitude response to variation in azimuth is an indication of multiple crack systems, where depending on whether the azimuthal direction is parallel or perpendicular to the crack system, the amplitude increases or decreases, respectively (Lynn 2014). This observation characterises an orthorhombic or higher symmetry media with multiple sets of aligned vertical cracks (Lynn 2014; Crampin 1993; Wild and Crampin 1991). The second significant seismic reflection amplitude anisotropy observed in the AVAZ result is a polarity reversal as the angle of incidence increases from near $\left(\leq 15^{\circ}\right)$ to mid offset $\left(\geq 30^{\circ}\right)$. The observed polarity reversal is attributed to the fact that $\mathrm{P}$-wave intersects embedded fractures at low angle azimuthal directions but propagates parallel at high angle azimuthal directions $\left(>50^{\circ}\right)$. Lynn

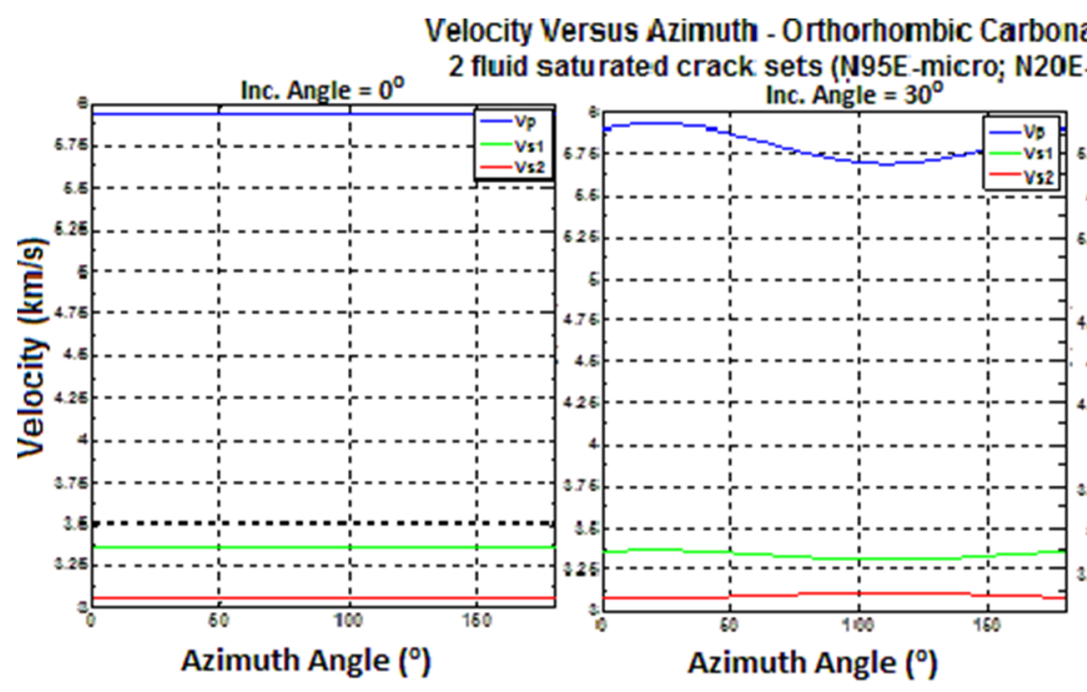

(a) (b)

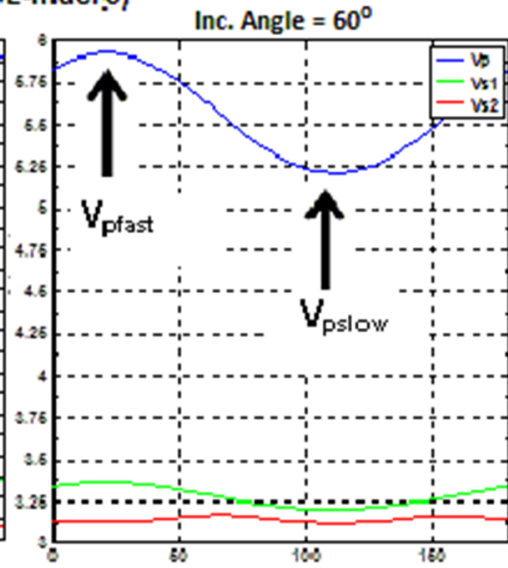

Azimuth Angle ( $\left.{ }^{\circ}\right)$

(c)

Figure 13

Velocity versus azimuth for orthorhombic medium (carbonate reservoir with oil saturated cracks) at $0^{\circ}, 30^{\circ}$ and $60^{\circ}$ angles of incidence 


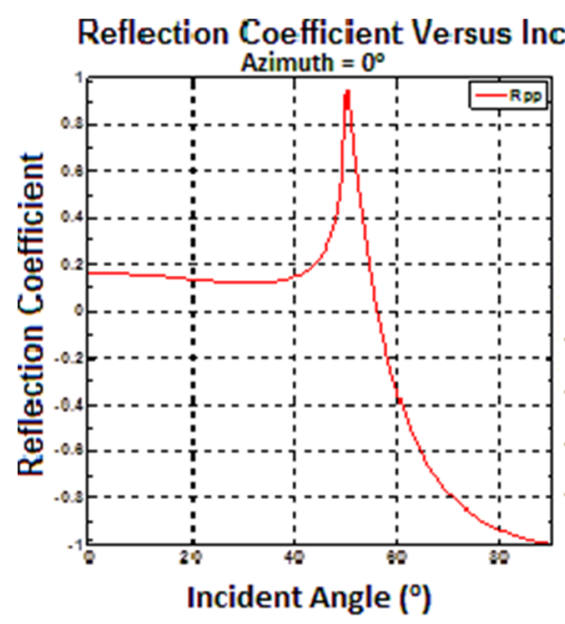

(a)

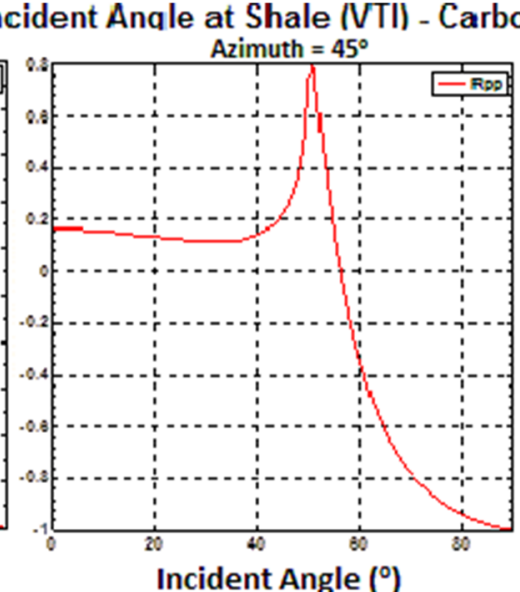

(b)

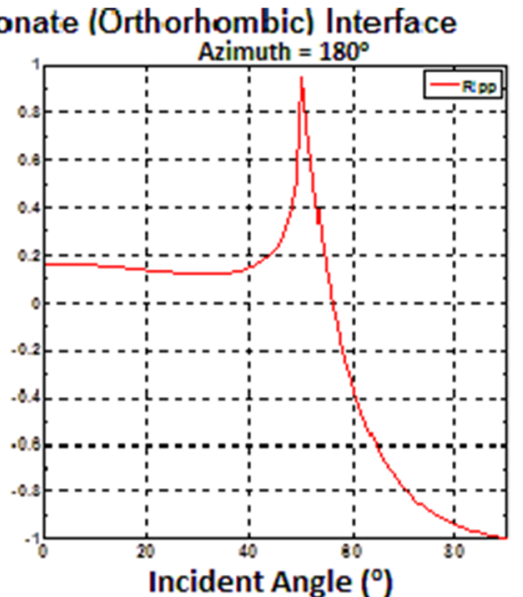

(c)

Figure 14

Variation of seismic reflection amplitude with incident angle at shale cap rock (VTI)—carbonate reservoir (orthorhombic) interface at near, mid and far offsets

(2014) attributed this flip to orthorhombic nature of the carbonate reservoir, specifically due to the magnitude of VTI and HTI anisotropy in both the carbonate reservoir and the shale overburden.

Finally, the resultant velocity attribute, $V_{\mathrm{p}}$, from the Earth model was fitted to a velocity-ellipse curve (Fig. 16) for estimating the orientation of the open crack that will store and support the flow of oil. The orientation of the major axis of the fitted velocity ellipse as well as the magnitude of the major and minor ellipse axes allows the determination of the direction of alignment of crack. It also assisted in the determination of the average crack intensity, which has a linear relationship with the crack density within the modelled carbonate reservoir. The ellipse curve fitted to P-wave velocity data has its major axis oriented N25.6E and this represents the direction of $V_{\mathrm{p}}$-fast which according to Bratton et al. (2006) is parallel to the fracture system. This implies that the resultant cracks within the carbonate reservoir are aligned in N25.6E direction.

The resultant crack direction within the carbonate reservoir was then used to determine the direction of minimum in situ stress, which is perpendicular to the general orientation of cracks within the reservoir. The direction of minimum in situ stress, which was determined from the study to be N115.6E, represents the direction of minimum resistance within the carbonate reservoir and thus essential to guide effective and cheap hydraulic fracture stimulation (fracking). Fracking along this direction should be effective and should be achieved at cheaper cost since less energy would be required to generate hydrostatic force necessary to exceed formation pressure for fracture to open.

\section{Conclusions}

An indirect method was adopted to generate an Earth model consisting of fine-layered VTI shale and underlying orthorhombic naturally fractured carbonate reservoir. Seismic attributes such as P- and $\mathrm{S}$-wave velocities as well as seismic reflection amplitude were extracted from the model. Both the velocity and amplitude attributes confirmed the occurrence of crack-induced azimuthal anisotropy in the carbonate reservoir. Azimuthal anisotropy is presented as variation in velocity with angle of incidence and azimuth, where the P-wave velocity is maximum $\left(V_{\mathrm{p}}\right.$-fast) in direction parallel to the crack and minimum $\left(V_{\mathrm{p}}\right.$-slow) in direction perpendicular to it. Azimuthally induced shear wave splitting into two vertically propagating $S_{1}$ and $S_{2}$ waves also 

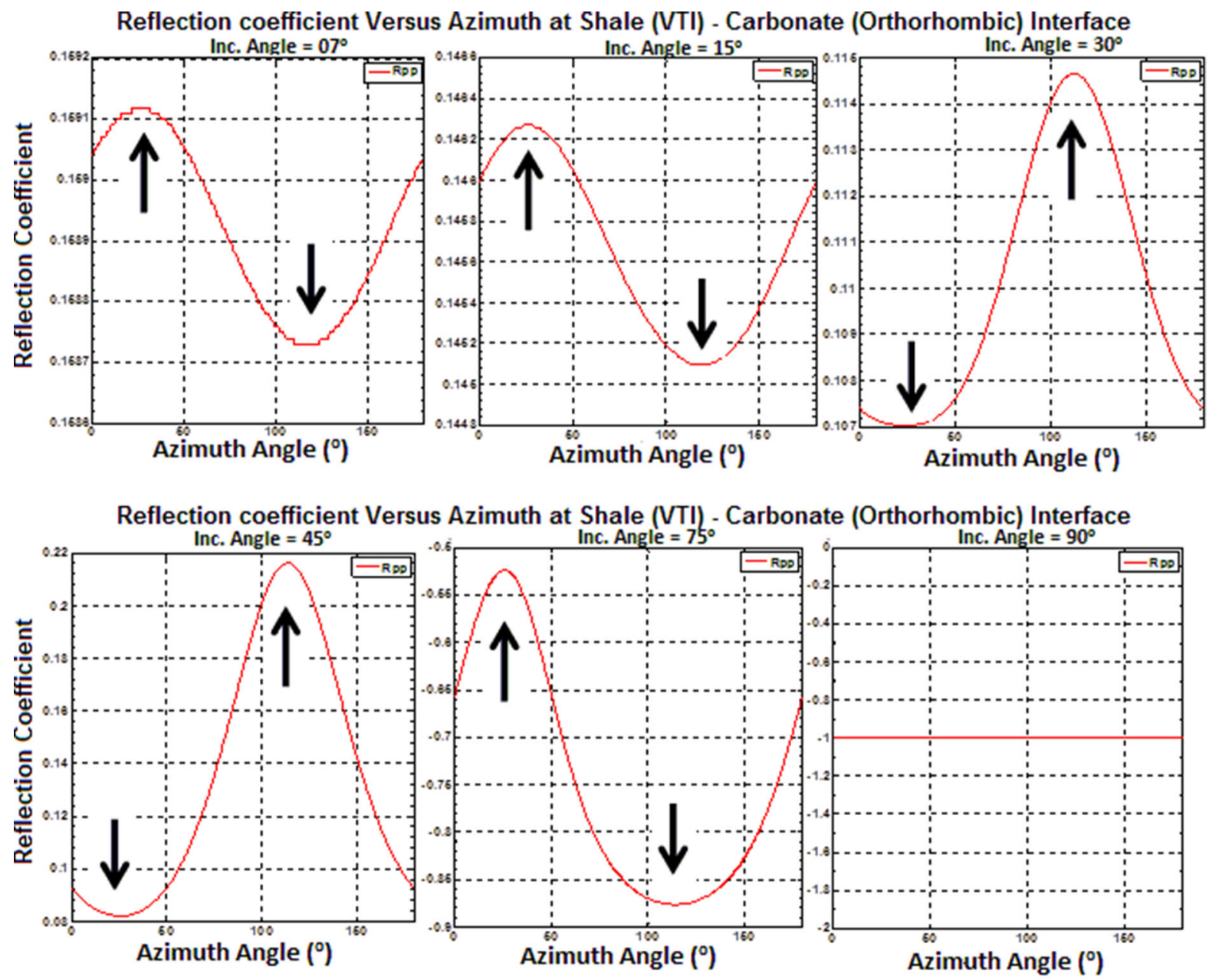

Bright Amplitude Azimuth Directions

Dim Amplitude Azimuth Directions

Figure 15

a-f Variation of seismic reflection amplitude with azimuth at shale (VTI)—carbonate reservoir (orthorhombic) interface at $07^{\circ}, 15^{\circ}, 30^{\circ}, 45^{\circ}$, $75^{\circ}$ and $90^{\circ}$ angle of incidence

established the occurrence of stress-induced aligned vertical cracks. The seismic reflection amplitude established the occurrence of azimuthal anisotropy which suggests the presence of stress-induced fluidsaturated vertical cracks. AVA indicates a classical class I AVO, while AVAZ did not only confirmed the occurrence of azimuthal anisotropy due to aligned vertical cracks, but also established a multi-crack case within the carbonate reservoir in the form of non-steady increase in azimuthal anisotropy with incident angle. This is also accompanied by a polarity reversal of bright-amplitude to dim-amplitude azimuth directions from near to far offset angles. This pattern has been identified through this modelling exercise to be related to the occurrence of multiple stress-induced aligned vertical cracks which defines a clear case of orthorhombic or higher symmetry. Finally, P-wave velocity ellipse curve fitting established the orientation of the ellipse axis which corresponds to the orientation of the macro-cracks. 


\section{Ellipse of P-wave Velocity in Orthorhombic Medium}

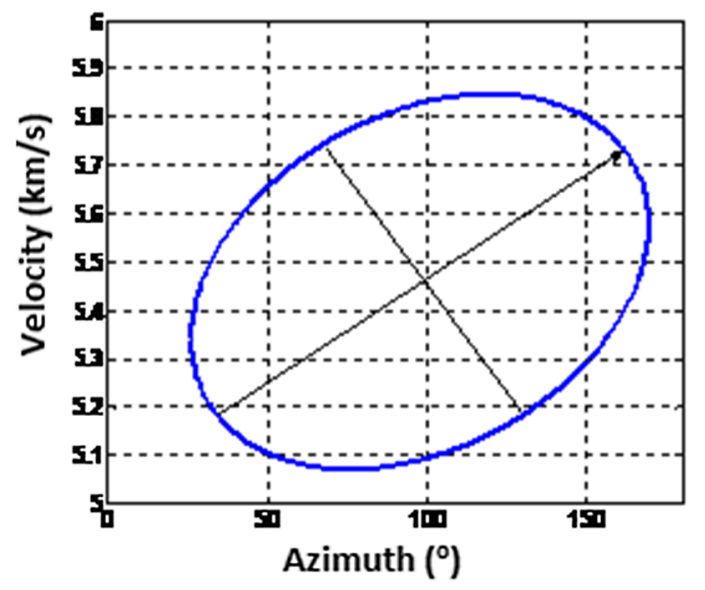

Figure 16

P-velocity-ellipse for determination of resultant crack orientation and crack intensity

\section{Acknowledgements}

This work was initiated by Lynn Incorporated, Colorado, USA and supported by British Geological Survey (BGS) and Edinburg Anisotropy Project (EAP). Dr. Hengchang Dai and Dr. Xiaoyang Wu are appreciated for offering many insightful suggestions. The invaluable suggestions by anonymous reviewers are highly acknowledged.

Open Access This article is distributed under the terms of the Creative Commons Attribution 4.0 International License (http:// creativecommons.org/licenses/by/4.0/), which permits unrestricted use, distribution, and reproduction in any medium, provided you give appropriate credit to the original author(s) and the source, provide a link to the Creative Commons license, and indicate if changes were made.

\section{REFERENCES}

Adams, C. (2003). Barnett Shale: A significant gas resource in the Fort Worth Basin. Texas: AAPG Southwest Section Annual Meeting.

Aki, K., \& Richards, P. G. (1980). Quantitative seismology: Theory and methods. San Francisco: W.H. Freeman and Co.

Alkhalifah, T., \& Tsvankin, I. (1995). Velocity analysis for transversely isotropic media. Geophysics, 60, 1550-1566.

Ball, M. M. (1995). Permian Basin Province (044). In D. L. Gautier, G. L. Dolton, K. I. Takahashi, \& K. L. Varnes (Eds.), National assessment of United States oil and gas resourcesResults, methodology, and supporting data. Texas: U.S. Geological Survey.
Bates, C. R., Lynn, H. B., \& Simon, M. (1999). The study of a natural fractured gas reservoir using seismic techniques. $A A P G$ Bulletin, 83, 1392-1407.

Bratton, T., Canh, D. V., Que, N. V., Duc, N. V., Gillespie, P., Hunt, D., et al. (2006). The nature of naturally fractured reservoirs. Oilfield Review, 18(2), 4-23.

Brown, J. S., \& Korringa, J. (1975). On the dependence of the elastic properties of a porous rock on the compressibility of the pore fluid. Geophysics, 40, 608-616.

Bush, I., \& Crampin, S. (1991). Paris basin VSPs: case history establishing combinations of matrix- and crackanisotropy from modelling shear wavefields near point singularities. Geophysical Journal International, 107, 433-447.

Calvert, A., Jenner, E., Jefferson, R., Bloor, R., Adams, N., \& Ramkhelawan, R. 2008. Preserving azimuthal velocity information: experiences with cross-spread noise attenuation and Offset Vector Tile (OVT) PreSTM. In: 78th Annual International Meeting. Las Vegas, SEG, pp. 207-211

Chapman, M. (2003). Frequency-dependent anisotropy due to meso-scale fractures in the presence of equant porosity. Geophysical Prospecting, 51, 369-379.

Childs, C., Walsh, J. J., \& Waterson, J. (1990). A method for estimation of the density of fault displacements below the limits of seismic resolution in reservoir formations. In A. T. Buller (Ed.), North Sea oil and gas reservoirs II (pp. 309-318). London: Graham and Trotman.

Crampin, S. (1981). A review of wave motion in anisotropic and cracked elastic media. Wave Motion, 3, 343-391.

Crampin, S. (1984). Anisotropy and transverse isotropy. First Break, 2, 19-21.

Crampin, S. (1985). Evaluation of anisotropy by shear-wave splitting. Geophysics, 50, 142-152.

Crampin, S. (1989). Suggestions for a consistent terminology for seismic anisotropy. Geophysical Prospecting, 37, 753-770.

Crampin, S. (1993). A review of the effects of crack geometry on wave propagation through aligned cracks. Canadian Journal of Exploration Geophysics, 29, 3-17.

Crampin, S., \& Peacock, S. (2008). A Review of the current understanding of shear-wave splitting in the crust and common fallacies in interpretation. Wave Motion, 45, 675-722.

Darwin, R. (1991). Roadside geology of Texas. Missoula, MT: Mountain Press.

Dvorkin, J., Mavko, G., \& Nur, A. (1995). Squirt flow in fully saturated rocks. Geophysics, 60, 97-107.

Dvorkin, J., Nolen-Hoeksema, R., \& Nur, A. (1994). The Squirt flow mechanism: macroscopic description. Geophysics, 59, 428-438.

Finkbeiner, T., Barton, C., \& Zoback, M. (1997). Relationships among in situ stress, fractures and faults, and fluid flow: Monterey formation, Santa Maria basin, California. AAPG Bulletin, 81, 1975-1999.

Friedman, M., \& Wiltschko, D. V. (1992). An approach to exploration for naturally fractured reservoirs, with examples from the Austin Chalk. In J. W. Schmoker, E. B. Coalson, \& C. A. Brown (Eds.), Geological studies relevant to horizorital drilliing: Examples from Westen North America (pp. 143-152). Texas: Rocky Mountain Association of Geologists.

Grechka, V., Mateeva, A., Franco, G., Gentry, C., Jorgensen, P., \& Lopez, J. (2007). Estimation of seismic anisotropy from P-wave VSP data. The Leading Edge, 26(6), 756-759. 
Helbig, K. (1994). Foundations of Anisotropy for Exploration Seismics. Handbook of Geophysical exploration. SEG. Oxford: Pergamon Press.

Hornby, B. E., Schwartz, L. M., \& Hudson, J. A. (1994). Anisotropic effective-medium modeling of the elastic properties of shales. Geophysics, 59, 1570-1583.

Hsu, C. J., \& Schoenberg, M. (1993). Elastic waves through a simulated fracture medium. Geophysics, 58, 964-977.

Hudson, J. A. (1981). Wave speeds and attenuation of elasticwaves in material containing cracks. Geophysical Journal International, 64, 133-150.

Lynn, H. B. 2014. Field data evidence of orthorhombic media: changes in the P-P bright azimuth with angle of incidence, Extended Abstract. Denver, SEG

Lynn, H. B., \& Thomsen, L. A. (1990). Reflection shear wave data collected near the principal axes of azimuthal anisotropy. Geophysics, 55, 147-156.

Mavko, G., \& Jizba, D. (1991). Estimating grain-scale fluid effects on velocity dispersion in rocks. Geophysics, 56, 1940-1949.

Montgomery, S. L., Jarvie, D. M., Bowker, K. A., \& Pollastro, R. M. (2005). Mississippian Barnett Shale, Fort Worth Basin: Northcentral Texas: Gas-shale play with multi-tcf potential. AAPG Bulletin, 89, 155-175.

Muller, T. M., Gurevich, B., \& Lebedev, M. (2010). Seismic wave attenuation and dispersion resulting from wave-induced flow in porous rocks: A review. Geophysics, 75(26), 260000.

Musgrave, M. J. P. 1970. Crystal acoustics: Holden Day.

Ross, A. M. (1970). Geologic and Historic Guide to the State Parks of Texas. Austin: Bureau of Economic Geology, University of Texas at Austin.

Rutherford, S. R., \& Williams, M. W. (1989). Amplitude-versus offset variations in gas sands. Geophysics, 54, 680-688.

Sayers, C. M. (2002). Stress-dependent elastic anisotropy of sandstones. Geophysical Prospecting, 50, 85-95.

Schoenberg, M. (1980). Elastic wave behaviour across linear slip interfaces. Journal of the Acoustical Society of America, 68, 1516-1521.
Schoenberg, M., \& Helbig, K. (1997). Orthorhombic media: Modeling elastic wave behavior in a vertically fractured earth. Geophysics, 62(6), 1954-1974.

Schoenberg, M., \& Sayers, C. M. (1995). Seismic anisotropy of fractured rock. Geophysics, 60(1), 204-211.

Stockwell, J. W., \& Cohen, J. K. (2008). The new SU user's manual. Colorado: Center for Wave phenomena, Colorado School of Mines.

Taner, M. T., \& Koehler, F. (1969). Velocity spectra digital computer derivation and applications of velocity functions. Geophysics, 34, 859-881.

Thomsen, L. (1986). Weak elastic anisotropy. Geophysics, 51, 1954-1966.

Thomsen, L. (1995). Elastic anisotropy due to aligned cracks in porous rock. Geophysical Prospecting, 43, 805-829.

Thomsen, L. (2002). Understanding seismic anisotropy in exploration and exploitation. Tulsa: Society of Exploration Geophysicists.

Tsvankin, I. (2001). Seismic signatures and analysis of reflection data in anisotropic media (3rd ed.). Colorado: Elsevier Science.

Tsvankin, I. (2005). Seismic signatures and analysis of reflection data in anisotropic media. Oxford: Elsevier.

Tsvankin, I., and V. Grechka, 2011, Seismology of azimuthally anisotropic media and seismic fracture characterization. Geophysical Reference Series 17, Society of Exploration Geophysicists.

Tsvankin, I., \& Thomsen, L. (1994). Nonhyperbolic reflection moveout in anisotropic media. Geophysics, 59, 1290-1304.

Wild, P., \& Crampin, S. (1991). The range of effects of azimuthal isotropy and EDA-anisotropy in sedimentary basins. Geophysical Journal International, 107, 513-529.

Yardley, G. S., \& Crampin, S. (1991). Extensive-dilatancy anisotropy: Relative information in VSPs and reflection surveys. Geophysical Prospecting, 39, 337-355. 